\title{
Modifications de l'OPAS, de la LiMA et du laboratoire au cabinet
}

\author{
Susanne Christena, Thomas Kessler ${ }^{b}$ \\ ${ }^{a}$ Dr méd., experte, division Médecine et tarifs ambulatoires; ${ }^{b}$ Expert, division Médecine et tarifs ambulatoires
}

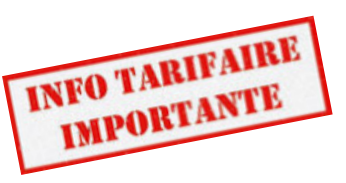

1 Ordonnance sur les prestations de l'assurance des soins.

2 Liste des moyens et appa reils.

\section{Remarque}

Au moment de rédiger cet article, seule la version provisoire des modifications de la LA, de l'OPAS et de la LiMA était connue. L'OFSP a annoncé la publication des modifications définitives pour le 12 ou 19 décembre 2017. Des divergences sont donc possibles; nous vous recommandons par conséquent de consulter le site internet de l'OFSP.

Correspondance: FMH / Division Médecine et tarifs ambulatoires Baslerstrasse 47

CH-4600 Olten

Tél. 0313591230 Fax 0313591238

tarife.ambulant[at]fmh.ch

\section{OPAS ${ }^{1}$, modifications au $1^{\text {er }}$ janvier 2018}

Pour toutes les positions de l'OPAS pour lesquelles il est fait référence à la prise en charge par l'assureur sous "Conditions», le texte standard suivant est introduit: «Les prestations ne sont prises en charge que si l'assureur-maladie donne préalablement une garantie spéciale en tenant compte de la recommandation du médecin-conseil.»

Les autres modifications concernent les thèmes suivants:

1. Vaccinations prophylactiques et examens de dépistage:

- Vaccination contre le papillomavirus humain (HPV): suppression du délai

- Dépistage chez les nouveau-nés: complété par l'acidurie glutarique de type 1 et la maladie du sirop d'érable

- Dépistage du cancer du côlon: aucune franchise n'est perçue pour cette prestation également dans le canton de Genève

2. Suppression de la position ou de l'obligation de prise en charge pour:

- Insulinothérapie à l'aide d'une pompe à perfusion continue

- Stimulation magnétique transcrânienne répétitive (rTMS) pour le traitement de la dépression

3. Adaptation des indications et des conditions pour:

- Greffe de cellules souches hématopoïétiques

- Traitement photodynamique à l'acide 5-aminolévulinique

- Système implantable pour l'enregistrement d'un électrocardiogramme sous-cutané

- Tomographie par émission de positrons (TEP, TEP/TC)

- Irradiation thérapeutique par faisceau de protons

4. Introduction d'un délai pour:

- Radiothérapie stéréotaxique ambulatoire (photons) de la forme exsudative de la dégénérescence maculaire liée à l'âge: limitée au 30.6.2020

Vous trouverez de plus amples informations sur le site de l'OFSP: https://www.bag.admin.ch/bag/fr/home/ service/gesetzgebung/gesetzgebung-versicherungen/ gesetzgebung-krankenversicherung/kvg.html

\section{LiMA ${ }^{2}$, modifications au $1^{\mathrm{er}}$ janvier 2018}

Les changements concernent les chapitres suivants:

- 03. Moyens d'application

- 09. Appareils d'électrostimulation

- 10. Accessoires de marche

- 14. Appareils d'inhalation et de respiration

- 21. Appareils de mesure des états et des fonctions de l'organisme

- 24. Prothèses

- 34. Matériel de pansement A

- 35. Matériel de pansement B

La plupart des modifications concernent les deux domaines générateurs des plus gros chiffres d'affaires, à savoir le matériel de pansement et les appareils de mesure pour diabétiques.

Vous trouverez de plus amples informations sur le site de l'OFSP: https://www.bag.admin.ch/bag/fr/home/themen/ versicherungen/krankenversicherung/krankenversiche rung-leistungen-tarife/Mittel-und-Gegenstaendeliste.html

\section{Laboratoire au cabinet}

Désormais, la position TARMED 00.0715 Ponction veineuse pour prise de sang par du personnel non médical ne doit pas uniquement avoir lieu dans le cadre du diagnostic en présence du patient mais peut aussi être facturée si l'analyse s'effectue dans un laboratoire extérieur. La réglementation transitoire relative à l'obligation d'obtenir l'AFC pour la pratique du laboratoire au cabinet médical (CMPR) échoit cette année. La participation régulière aux contrôles de qualité externes (CQE) correspond à une recertification. La CMPR n'établit donc aucun certificat séparé; la preuve de la participation aux CQE suffit. La liste des analyses soumises au contrôle de qualité externe obligatoire est adaptée chaque année et publiée sur le site de la QUALAB (www.qualab.ch).

Liste des analyses (LA), modifications au $1^{\mathrm{er}}$ janvier 2018: à l'heure actuelle, aucun changement ne concerne le diagnostic en présence au laboratoire du cabinet. Vous trouverez de plus amples informations sur la LA sur le site de l'OFSP: https://www.bag.admin.ch/bag/fr/home/ themen/versicherungen/krankenversicherung/kranken versicherung-leistungen-tarife/Analysenliste.html 